Def ect $i$ on- sel ect $i$ ve sol ubi I i zat $i$ on and chemi cal I y-responsi ve sol ubi I ity swi tching of si ngl e- wal l ed carbon nanot ubes wi th cucur bi t [ 7] uri I

\begin{tabular}{|l|l|}
\hline 著者 & $\begin{array}{l}\text { Ogoshi Tonøki, I nagaki Ayumi, Yanagi shi } \\
\text { Tada- aki, Nakanmt o Yoshi aki }\end{array}$ \\
\hline 著者別表示 & 生越 友樹, 山岸 忠明, 中本 義章 \\
\hline $\begin{array}{l}\text { j our nal or } \\
\text { publ i cat i on ti t l e }\end{array}$ & Chemi cal Communi cat i ons \\
\hline vol une & 19 \\
\hline page range & $2245-2247$ \\
\hline year & 2008 01-01 \\
\hline URL & ht t p: //doi . or g/10. 24517/00011028 \\
\hline
\end{tabular}




\title{
Defection-Selective Solubilization and Chemically-Responsive Solubility Switching of Single- Walled Carbon Nanotubes with Cucurbit[7]uril
}

\author{
` Tomoki Ogoshi*, Ayumi Inagaki, Tada-aki Yamagishi and Yoshiaki Nakamoto* \\ Receipt/Acceptance Data [DO NOT ALTER/DELETE THIS TEXT] \\ Publication data [DO NOT ALTER/DELETE THIS TEXT] \\ DOI: 10.1039/b000000x [DO NOT ALTER/DELETE THIS TEXT]
}

Single-walled carbon nanotubes (SWCNTs) were suspended in 10 aqueous media with cucurbit[7]uril (CB7), while SWCNTs were insoluble with cucurbit[5]uril (CB5). Moreover, defectionselective solubilization of SWCNTs with $\mathrm{CB} 7$ was demonstrated.

Single-walled carbon nanotubes (SWCNTs) are onedimensional nanomaterials with unusual properties and ${ }_{15}$ potential applications. ${ }^{1}$ Especially, for the application of SWCNTs in biology and material science, the preparation of water-soluble SWCNTs has been attractive research target. Solubilization of SWCNTs in aqueous media using amphiphilic polymers ${ }^{2}, \mathrm{DNA}^{3}$, peptides ${ }^{4}$ and surfactants ${ }^{5}$ has 20 been reported. Solubility switching of SWCNTs in response to environmental triggers, such as $\mathrm{pH}$ change $^{6}$, ions ${ }^{7}$, chemicals $^{8}$ and light ${ }^{9}$, is also intriguing and important for the applications of SWCNT-based sensors, because electrical and optical properties of SWCNTs are extremely sensitive to the ${ }_{25}$ dispersion state of SWCNTs. Moreover, solubilization of SWCNTs aimed toward their purification in terms of diameters and defections is technologically important, since there have so far been no methods for selective preparation of SWCNTs with narrow distribution diameters and no 30 defections.

Our current interest is preparation of water-soluble SWCNTs using macrocyclic host molecules. ${ }^{8,10,11}$ Because host compounds form host-guest complexes with various guests, host molecules around SWCNT are able to capture 35 guest molecules on SWCNT surface and solubilize the SWCNT simultaneously. Herein, we report on solubilization of SWCNTs in aqueous media by using hosts of cucurbit[n]urils (CBs) as solubilizing agent. Since CBs captured various guests into their cavity ${ }^{12}$ and preferably 40 formed 2:1 host-guest complex with $\mathrm{C}^{13} 0^{13}$, in the present research, we investigated solubilization of SWCNTs with CBs. CBs $[n=5$ (CB5) and 7 (CB7)] were employed as solubilizing agent of SWCNTs (Fig. 1(A)). Interestingly, by using CBs as

Department of Chemistry and Chemical Engineering, Graduate School of Natural Science and Technology, Kanazawa University, Kakuma-machi, Kanazawa 920-1192, Japan

E-mail: ogoshi@t.kanazawa-u.ac.jp; Fax+81 76-234-4775; Tel+8176$234-4800$

$\uparrow$ Electronic Supplementary Information (ESI) available: Experimental section, UV-Vis spectra of soluble Hipco SWCNTs with the increasing of CB7 concentration, UV-Vis spectra of Hipco SWCNTs suspended in CB7 and SDBS, photo and UV-Vis spectra of CB7/Hipco SWCNT hybrids upon addition of $\mathrm{AdNH}_{2},{ }^{1} \mathrm{H}$ NMR spectra of the supernatant after addition of $\mathrm{AdNH}_{2}, \mathrm{UV}-\mathrm{V}$ is spectra of the supernatants of CoMoCAT and CarboLex SWCNTs with CB7, and Raman spectra of pristine Hipco SWCNTs and SWCNT-COOH.
(A)

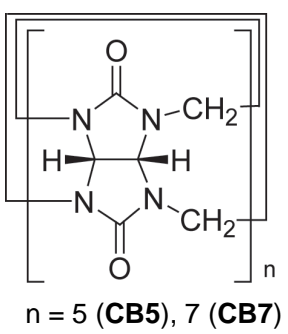

(B)

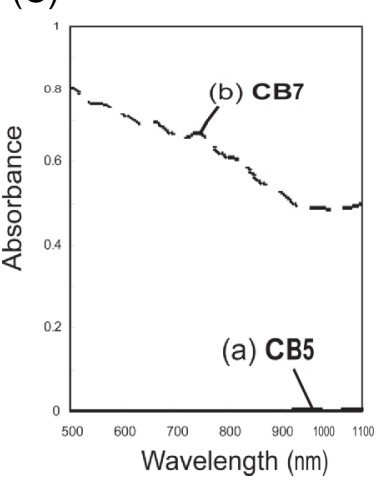

(a) CB5

(b) CB7

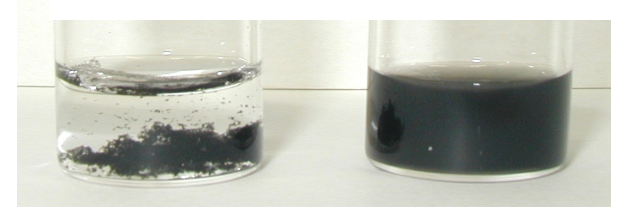

Fig. 1 (A) Chemical structure of cucurbiturils. (B) Photos of Hipco SWCNTs in aqueous media with (a) CB5 and (b) CB7 after sonication. (C) UV-Vis spectra of aqueous supernatants $(5 \mathrm{~mL})$ containing Hipco SWCNTs (1 mg) with (a) CB5 (solid line) and (b) CB7 (dash line) after sonication.

solubilizing agent, solubility of SWCNTs in aqueous media 45 clearly depended on cavity size of CBs, addition of guests and salts. Moreover, CBs showed defection-selective solubilization of SWCNTs.

We purchased Hipco SWCNTs from Carbon nanotechnologies, Inc., Texas, USA. The Hipco SWCNTs ${ }_{50}$ were purified according to previous paper described. ${ }^{14} \mathrm{We}$ used CB5 and CB7 as solubilizing agent because of high solubility of CB5 and CB7 in water (water-solubility is about 20-30 mM). To suspension of Hipco SWCNTs $(1.0 \mathrm{mg})$ in aqueous solution $(5.0 \mathrm{~mL})$, solubilizer $(20 \mathrm{mg})$ was added and ${ }_{55}$ the resulting solution was sonicated for $3 \mathrm{~h}$ at room temperature. In case of $\mathbf{C B 7}$, during the sonication, the aqueous solution changed from colorless to black, indicating solubilization of Hipco SWCNTs with CB7 (Fig. 1(B)(b)). After the sonication, insoluble Hipco SWCNTs (ca. $0.80 \mathrm{mg}$ ) 60 were removed by centrifugation $(12500 \mathrm{~g})$. The supernatant using CB7 was homogeneous black solution and extremely stable for more than a month. In contrast, in the presence of CB5, Hipco SWCNTs were insoluble even after sonication 


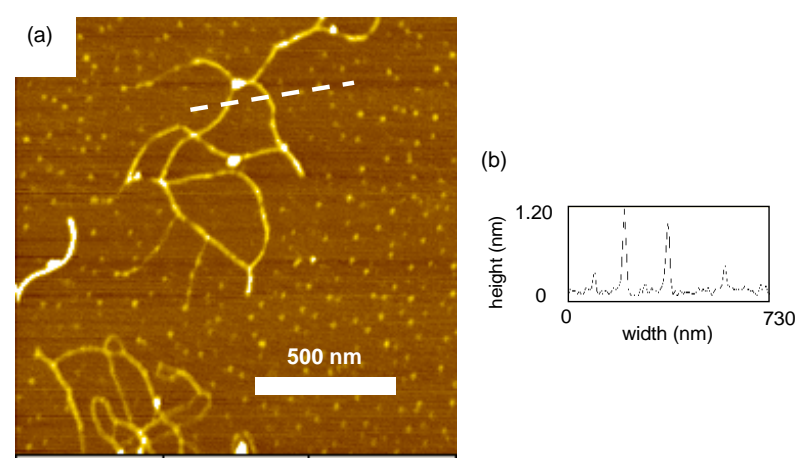

Fig. 2 (a) Tapping mode atomic force microscopic image of CB7/Hipco SWCNT hybrids. (b) Height profile along dash line in (a).

(Fig. 1(B)(a)). Fig. 1(C) shows UV-Vis spectra of the ${ }_{65}$ supernatants after centrifugation. In the region of $500-900$ nm, typical Hipco SWCNT van Hove singularities were observed in the presence of CB7 (Fig. 1(C)(b)), while no absorption bands were found in the presence of CB5 (Fig. 1(C)(a)). From these data, Hipco SWCNTs were soluble in 70 aqueous solution with CB7 and insoluble with CB5. Solubilization ability of CB7 was compared to that of conventional solubilizer of sodium dodecylbenzenesulfonate (SDBS). ${ }^{5}$ Solubility of Hipco SWCNTs with CB7 was higher than that with SDBS (ESI). Solubility of Hipco SWCNTs with ${ }_{75}$ CB7 (4 mg mL ${ }^{-1}$ ) was $3.42 \times 10^{-2} \mathrm{mg} \mathrm{mL}^{-1}$. We examined effect of the concentration of $\mathbf{C B} 7$ on solubility of Hipco SWCNTs in aqueous media. As the concentration of CB7 increased, solubility of Hipco SWCNTs also increased (ESI).

From tapping mode atomic force microscopic (TM-AFM) ${ }_{80}$ image of Hipco SWCNTs solubilized by CB7 (CB7/Hipco SWCNT hybrids), nanotubes were observed (Fig. 2(a)) and average size of the tubes was about $1.1-1.2 \mathrm{~nm}$ (Fig. 2(b)). Since the average diameter of Hipco SWCNT is $0.8-1.2 \mathrm{~nm}^{15}$, the nanotube observed is individual SWCNT.

85 Solubilization of Hipco SWCNTs with host-guest complex was carried out. 1-Adamantanamine $\left(\mathrm{AdNH}_{2}\right)$ was used as guest. With the mixture of $\mathbf{C B 7}$ and $\mathrm{AdNH}_{2}$, Hipco SWCNTs were insoluble in aqueous solution. When $\mathrm{AdNH}_{2}$ was added to CB7/Hipco SWCNT hybrids, aggregation of Hipco ${ }_{90}$ SWCNTs was observed (ESI). These observations indicate that Hipco SWCNTs were insoluble by formation of hostguest complex with $\mathrm{AdNH}_{2} \cdot{ }^{16}$ Furthermore, upon addition of aqueous $\mathrm{NaCl}$ or hydrochloric acid solution, precipitation of Hipco SWCNTs was also observed. Because urea groups of ${ }_{95}$ CB7 bind cations via ion-dipole interaction ${ }^{12}$, CB7 binds sodium cation and proton as guest. From these data, it was found that complex between CB7 and Hipco SWCNT was dissociated by formation of $\mathbf{C B 7}$-guest complexes. The binding of CB7 with Hipco SWCNT is weaker than that of ${ }_{100}$ CB7-guest complexes.

From these observations, we examine nanostructure of CB7/Hipco SWCNT hybrids. Since Hipco SWCNTs were insoluble with CB5 and soluble with CB7, cavity size of CBs should effect on solubility of Hipco SWCNTs in aqueous 105 solution. Moreover, by adding guests such as $\mathrm{AdNH}_{2}$ and cation, aggregation of Hipco SWCNTs was observed, also indicating that cavity of $\mathbf{C B} 7$ should act as an important role for solubilization of Hipco SWCNTs. However, considering
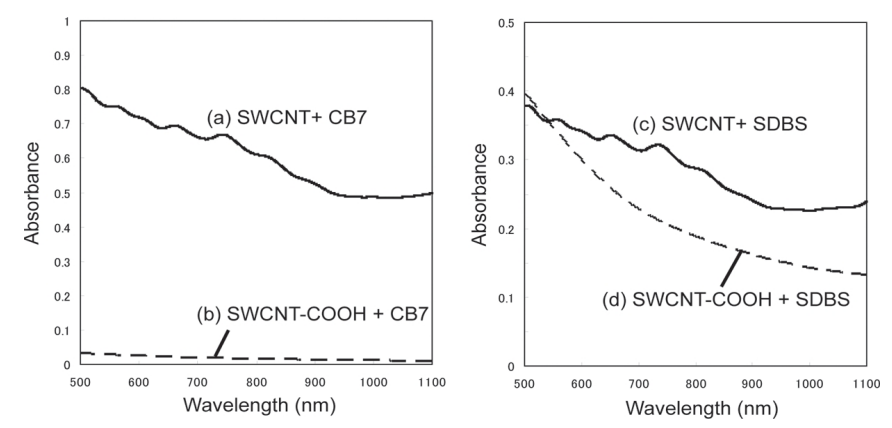

Fig. 3 UV-Vis spectra of the supernatants of (a) pristine Hipco SWCNTs with CB7 (solid line), (b) SWCNT-COOH with CB7 (dash line), (c) pristine Hipco SWCNTs with SDBS (solid line) and (d) SWCNT-COOH with SDBS (dash line) after sonication.

that the portal size of $\mathbf{C B} 7$ cavity is about $0.54 \mathrm{~nm}^{12}$ and the 110 outer diameter of Hipco SWCNT is about $1.0 \mathrm{~nm}^{15}$, cavity of CB7 is too small to form pseudo-rotaxane structure between Hipco SWCNT as axle and CB7 as ring. By using CoMoCAT SWCNTs $^{17}$ (average diameter $=0.8 \mathrm{~nm}$ ) and CarboLex SWCNTs (average diameter $=1.3 \mathrm{~nm}$ ) instead of Hipco 115 SWCNTs, CoMoCAT and CarboLex SWCNTs were watersoluble with CB7 (ESI). The data indicate that solubilization of SWCNTs with CB7 is independent of diameter of SWCNTs. Thus, SWCNTs are not solubilized by forming pseudo-rotaxane structure. The other possible solubilization 120 mechanism should be adsorption of CB7 on SWCNT surface. Amphiphilic urea groups of $\mathbf{C B 7}$ might be adsorbed to SWCNT surface. Generally, amphiphilic polymers such as $\operatorname{poly}(\mathrm{N}$-vinyl-2-pyrrolidone) and poly(ethylene glycol) are able to solubilize SWCNTs in aqueous media by wrapping. ${ }^{2}$ 125 Moreover, for the nonionic surfactants such as Triton X-405, poly( $N$-vinyl-2-pyrrolidone) and poly(ethylene glycol), surfactants with high molecular weight were able to suspend more SWCNTs. ${ }^{5}$ Therefore, in the same way as amphiphilic polymers, CB7 might be easily adsorbed to SWCNT surface ${ }_{130}$ compared to $\mathbf{C B 5}$. The same trends were also observed in solubilization of SWCNTs with water-soluble amphiphilic calixarenes. $^{10}$

By using CB7 as solubilizing agent, solubilization of Hipco SWCNTs with defection sites on graphitic surface and at ends 135 was examined. By treating with boiling diluted $2 \mathrm{M}$ nitric acid for $24 \mathrm{~h}$, acid cut SWCNT (SWCNT-COOH) was obtained. ${ }^{18}$ SWCNT-COOH was insoluble in aqueous solution by using CB7 as solubilizer, while pristine nondefective Hipco SWCNTs were soluble with CB7. In contrast, by using SDBS, 140 both Hipco SWCNTs and SWCNT-COOH were soluble in aqueous media. UV-Vis spectra of these supernatants are shown in Fig. 3. The absorption of SWCNT-COOH was not observed in the supernatant of SWCNT-COOH with CB7 (Fig. 3(b)), while typical Hipco van Hove singularities were 45 observed in Hipco SWCNTs with CB7 (Fig. 3(a)). In the supernatant containing SWCNT-COOH suspended with SDBS, absorption band in the region of $500-1100 \mathrm{~nm}$ was observed (Fig. 3(d)), indicating solubilization of SWCNT$\mathrm{COOH}$ with SDBS. The absorption displayed a loss of ${ }_{150}$ features compared to typical Hipco SWCNT van Hove singularities (Fig. 3(c)), suggesting a disruption in the electron structure due to oxidation of Hipco SWCNTs. ${ }^{19}$ From these 
observations, by using CB7 as a solubilizer, Hipco SWCNTs were water-soluble but defected SWCNT-COOH was 155 insoluble in water. Since typical surfactant of SDBS solubilizes both Hipco SWCNTs and defected SWCNT$\mathrm{COOH}$ and solubility of SWCNTs generally increases with increasing number of oxidation sites on $\mathrm{SWCNT}^{18}, \mathbf{C B} 7$ is able to selectively solubilize nondefective SWCNTs.

160 Formation of host-guest complex between $\mathbf{C B} 7$ and proton of carboxylic acid from SWCNT-COOH should result in the defection-selective insolubilization of SWCNTs.

In conclusion, by using CB7, water-soluble SWCNTs were successfully prepared. To the best of our knowledge, it is the

165 first example of solubilization of SWCNTs with CBs. CB7 wrapped SWCNT and solubility of SWCNTs clearly changed by adding guests. Moreover, CB7 was able to selectively solubilize nondefective SWCNTs in aqueous media. There are few examples of defection-selective solubilization of ${ }_{170}$ SWCNTs, while diameter selective solubilization of SWCNTs has been reported. ${ }^{20}$ Since oxidation damages of nanotubes lose valuable material property, selective ablation of defective SWCNTs is technologically important. Thus, CB7 will be used not only for solubilizer of SWCNTs but also for 175 purification of defected SWCNTs.

We thank Prof. Kohshin Takahashi (Kanazawa University) for TM-AFM measurements and Dr. Tomokazu Umeyama (Kyoto University) for many useful discussions. This work was supported by the Kinki Invention Center and a Grant-in180 Aid for Young Scientists (WAKATE B-1975011) from the ministry of Education, Culture, Sports, Science and Technology, Japan.

\section{Notes and references}

1 D. Tasis, N. Tagmatarchis, A. Bianco and M. Prato, Chem. Rev., 185 2006, 106, 1105; N. R. Baughman, A. A. Zakhidov and W. A. de Heer, Science, 2002, 297, 787; J. Chen, M. A. Hamon, H. Hu, Y. S. Chen, A. M. Rao, P. C. Eklund and R. C. Haddon, Science, 1998, 282, 95; J. Liu, A. G. Rinzler, H. J. Dai, J. H. Hafner, R. K. Bradley, P. J. Boul, A. Lu, T. Iverson, K. Shelimov, C. B. Huffman, F. Rodriguez-Macias, Y. S. Shon, T. R. Lee, D. T. Colbert and R. E. Smalley, Science, 1998, 280, 1253; V. Zorbas, A. O. Acevedo, A. B. Daltonm, M. M. Yoshida, G. R. Dieckmann, R. K. Draper, R. H. Baughman, M. J. Yacaman and I. H. Musselman, J. Am. Chem. Soc., 2004, 126, 7222; N. Nakashima, Y. Tomonari, H. Murakami and K. Yoshinaga, Chem. Lett., 2002, 31, 638.

2 M. J. O'Connell, P. Boul, L. M. Ericson, C. Huffman, Y. H. Wang, E. Haroz, C. Kuper, J. M. Tour, K. D. Ausman and R. E. Smalley, Chem. Phys. Lett., 2002, 364, 303; C. Park, Z. Ounaies, K. A. Watson, R. E. Crooks, J. Jr. Smith, S. E. Lowther, J. W. Connell, E. 200 J. Siochi, J. S. Harrison and T. L. St. Chair, Chem. Phys. Lett., 2002, 364, 303.

3 G. R. Dieckmann, A. B. Dalton, P. A. Johnson, J. Razal, J. Chen, G. M. Giordano, E. Muñoz, I. H. Musselman, R. H. Baughman and R. K. Draper, J. Am. Chem. Soc., 2003, 125, 1770.

2054 A. Ishibashi, Y. Yamaguchi, H. Murakami and N. Nakashima, Chem. Phys. Lett., 2006, 419, 574.

5 V. C. Moore, M. S. Strano, E. H. Haroz, R. H. Hauge, R. E. Smalley, J. Schmidt and Y. Talmon, Nano Lett., 2003, 3, 1379.

6 J. C. Grunlan, L. Liu and Y. S. Kim, Nano Lett., 2006, 6, 911; W.

210 Zhao, C. Song and P. E. Pehrsson, J. Am. Chem. Soc., 2002, 124, 12418.

7 B. Yu, F. Zhou, G. Liu, Y. Liang, W. T. S. Huck and W. Liu, Chem. Commun., 2006, 2356

8 T. Ogoshi, Y. Takashima, H. Yamaguchi and A. Harada, J. Am. 215 Chem. Soc., 2007, 129, 4878.
9 K. Narimitsu, J. Nishioka, H. Murakami and N. Nakashima, Chem. Lett., 2006, 35, 892.

10 T. Ogoshi, T. Yamagishi and Y. Nakamoto, Chem. Commun., 2007, 4776.

22011 T. Ogoshi, T. Yamagishi, Y. Nakamoto and A. Harada, Chem. Lett., 2007, 36, 1026

12 J. W. Lee, S. Samal, N. Selvapalam, H. J. Kim and K. Kim, Acc. Chem. Res., 2003, 36, 621; J. Lagona, P. Mukhopadhyay, S. Chakrabarti and L. Isaacs, Angew. Chem. Int. Ed., 2005, 44, 4844.

22513 F. Constabel and K. E. Geckeler, Tetrahedron Lett., 2004, 45, 2071.

14 W. Zhou, Y. H. Ooi, R. Russo, P. Papanek, D. E. Luzzi, J. E. Fischer, M. J. Bronikowski, P. A. Willis and R. E. Smalley, Chem. Phys. Lett., 2001, 350, 6.

15 P. Nikolaev, M. Bronikowski, R. Bradley, F. Rohmund, D. Colbert, K. Smith and R. E. Smalley, Chem. Phys. Lett., 1999, 313, 91.

16 Formation of the host-guest complex between $\mathbf{C B} 7$ and $\mathrm{AdNH}_{2}$ was checked by ${ }^{1} \mathrm{H}$ NMR measurements (ESI).

17 S. M. Bachilo, L. Balzano, J. E. Herrera, F. Pompeo, D. E. Resasco and R. B. Weisman, J. Am. Chem. Soc., 2003, 125, 11186.

23518 M. N. Tchoul, W. T. Ford, G. Lolli, D. E. Resasco and S. Arepalli, Chem. Mater., 2007, 19, 5765.

19 Defection of SWCNT-COOH was checked by Raman measurements (ESI).

20 S. Banerjee, T. H. Benny and S. S. Wong, J. Nanosci. Nanotechnol., 2005, 5, 841; S. Banerjee and S. S. Wong, J. Am. Chem. Soc., 2004, 126, 2073; Z. B. Zhang and S. L. Zhang, J. Am. Chem. Soc., 2007, 129, 666 . 
Single column figure/scheme (below)

$\mathrm{X}$

Fig./Scheme XX Caption.

Double column figure/scheme (below)

$\mathrm{X}$

Fig./Scheme XX Caption.

Single column image (no caption) (below)

$\mathrm{X}$

Double column image (no caption) (below)

$\mathrm{X}$

Single column numbered equation/reaction (below)

$\mathrm{X}$

(X)

Single column table (below)

Table XX Caption

$\mathrm{X}$

${ }^{a}$ Footnote text.

sou Double column table (below)

Table XX Caption

$\mathrm{X}$

${ }^{a}$ Footnote text. 